\title{
ANÁLISIS DIDÁCTICO DE POR QUÉ Y PARA QUÉ EDUCAMOS DENTRO DEL TRABAJO DE FIN DE MÁSTER DE UNA UNIVERSIDAD ESPAÑOLA
}

\section{DIDACTIC ANALYSIS OF WHAT FOR AND WHY WE TEACH WITHIN THE MATER'S FINAL PROJECT IN A SPANISH UNIVERSITY}

Antonio Nadal Masegosa ${ }^{1}$

\begin{abstract}
Departamento de Teoría e Historia de la Educación y Métodos de Investigación y Diagnóstico en Educación. Universidad de Málaga, Estado español.
\end{abstract}

\section{RESUMEN}

La legislación del Estado español sobre el Máster Universitario en Profesorado de Enseñanza Secundaria Obligatoria y Bachillerato, Formación

$1 \quad$ Licenciado y Doctor en Pedagogía (Universidad de Málaga, Estado español), Máster en Gestión de la Cooperación Internacional y de las ONGs (Universidad de Granada, Estado español). Grupo de Investigación HUM246 Cultura de la Diversidad y Escuela (Junta de Andalucía, Estado español). Profesor de la Facultad de Ciencias de la Educación de la Universidad de Málaga. Autor de La Escuela Moderna. Análisis histórico, publicado por la editorial de alto impacto La Muralla, y de 20 capítulos de libro hasta junio de 2020, con otros capítulos a la espera de publicación. antonionm@uma.es

Orcid: https://orcid.org/0000-0002-2788-0058 Google Scholar: https://scholar.google.es/citations?user=umR8u-EAAAAJ\&hl=es
Profesional y Enseñanza de Idiomas no incluye un gran desarrollo normativo sobre estos estudios, quedando al arbitrio de las facultades y universidades determinadas cuestiones académicas fundamentales. En menos de un año natural, se otorga la credencial al alumnado procedente de todo tipo de titulaciones sin relación alguna con la enseñanza o la pedagogía para que se titule como acreditado para la docencia en enseñanza secundaria o en formación profesional. Los trabajos de fin de máster y su presentación ante un tribunal son el último acto que deben desarrollar los y las estudiantes y futuros/as docentes. En el caso 
de la Universidad de Málaga, dichos trabajos incluyen la exigencia, de inicio, de responder a por qué y para qué educamos, una pregunta frente a la cual el alumnado en ocasiones no tiene la formación y los recursos suficientes para responder con solvencia.

PALABRAS CLAVE: Educación, Enseñanza, Formación de docentes de secundaria, Universidad.

\section{SUMMARY}

The legislation of the Spanish State on the University Master's Degree in Compulsory Secondary Education and Baccalaureate, Vocational Training and Language Teaching does not include a great normative development on these studies, leaving certain fundamental academic questions to the discretion of the faculties and universities. In less than a calendar year, the credential is awarded to students from all kinds of degrees unrelated to teaching or pedagogy so that they can be certified as teachers in secondary education or vocational training. The final master's projects and their presentation in front of a tribunal are the last act to be carried out by students and future teachers. In the case of the University of Malaga, these projects include the requirement, at its outset, to answer why and what we teach for, a question for which students sometimes do not have the training and enough resources to answer with solvency.

KEY WORDS: Education, Secondary Teacher Training, Teaching, University.

\section{RESUMO}

A legislação do Estado espanhol sobre o mestrado universitário em ensino médio obrigatório e bacharelado, treinamento vocacional e ensino de idiomas não inclui um grande desenvolvimento normativo nesses estudos, deixando certas questões acadêmicas fundamentais a critério das faculdades e universidades. Em menos de um ano civil, a credencial é concedida a estudantes de todos os tipos de graus não relacionados ao ensino ou pedagogia, para que possam ser certificados como ensino no ensino médio ou no treinamento vocacional. Os projetos finais de mestrado e sua apresentação em tribunal são o último ato a ser realizado por estudantes e futuros professores. No caso da Universidade de Málaga, esses trabalhos incluem a exigência, desde o início, de responder por que e para o que educamos, uma pergunta contra a qual os alunos às vezes não têm o treinamento e os recursos suficientes para responder com solvência. .

Palavras-chave: Educação, Ensino, Formação Secundária de Professores, Universidade.

\section{INTRODUCCIÓN}

Año tras año, para el profesorado que realmente lee los Trabajos de Fin de Máster (TFM) que debe autorizar para su presentación en tribunal -o ya en dicho acto oficial, calificar-, concretamente en el Máster en Profesorado de Enseñanza Secundaria Obligatoria, Bachillerato, Formación Profesional e Idiomas (MAES) en una universidad española -título habilitante-, es posible que sea un tanto monótono leer en ocasiones:

Se conoce con el término educar la actividad que consiste en transmitir determinados conocimientos y patrones de comportamiento con el fin de garantizar la continuidad de la cultura de la sociedad.

La palabra educar es de origen latín ducere que significa "guiar o conducir" en el conocimiento.

Educar consiste en enseñar a temprana edad valores, conocimientos, costumbres y formas de actuar, que permiten a un individuo vivir en sociedad. 
También, educar consiste en estimular, desenvolver y orientar aptitudes del individuo, de acuerdo con las ideas de una sociedad determinada.

La educación se lleva a cabo principalmente en los hogares, luego en las escuelas, las universidades, entre otras instituciones.

Asimismo, las instituciones deben de contar con un plan de estudios en el cual contemple [sic] la orientación del individuo a su desarrollo e integración plena en la sociedad (Significados. com, 2020).

Aún peor sería encontrar, como autorizados, es decir, ya en el trámite de presentación en el tribunal -incluso siendo miembro personal del mismo quien escribe-, Trabajos de Fin de Máster con los párrafos anteriores cortados y pegados, sin citar, es decir, plagiando.

\section{EL PROBLEMA DEL PLAGIO UNIVERSITARIO}

El plagio no existe, por ejemplo, como motivo de suspenso automático en la Plantilla de Evaluación de la Comisión Evaluadora en la Universidad de Málaga (2019a), en el Estado español, un documento público que llevaría sin modificación alguna desde el año 2015. El plagio ha sido denunciado en la Universidad de Málaga (Delgado, 2020), sin que hasta el momento conozcamos de forma pública consecuencias contundentes contra ello. Sí tenemos un alto número de casos en la universidad española, con algunas condenas (Chicote, 2020; Moreno, 2020; Ramajo, 2020; Villarreal et al., 2019). Pese a ello, durante el curso 2019/2020 personalmente sufriría un percance por escribir en un foro privado del citado Máster de la Universidad de Málaga que sabemos el tiempo que transcurre entre el final de las prácticas del MAES y la entrega de su memoria, y subir igualmente el TFM al campus virtual del MAES en la Facultad de Ciencias de la Educación (escaso tiempo: las prácticas se entregaban como máximo el 18 de mayo de 2020 a las 23.55; el TFM el 14 de junio a las 23.55 horas), especialmente teniendo en cuenta que hablamos de un próximo profesorado, a mi juicio, con nula preparación pedagógica (a veces con faltas de ortografía y expresión, y plagios, flagrantes). Creyendo que el artículo 20 de la Constitución Española me amparaba en dicha afirmación fundamentada en la práctica, sin embargo, sería invitado a retirar el comentario crítico, hecho que ejecuté, y la incidencia no tendría mayor recorrido. Lo que sí se evidenciaría es la incapacidad de iniciar un proceso contra el mal que implica el plagio universitario.

\section{LA FORMACIÓN PEDAGÓGICA BÁSICA}

Quienes estudiaron la Licenciatura 0 el Grado en Pedagogía tuvieron cuatro años de formación. Añadamos, en algunos casos, dos años más de doctorado, más los años de tesis doctoral. Busquemos, simplemente, el concepto pedagogía en las vigentes Plantilla de los tribunales para calificar TFM (Universidad de Málaga, 2019a) y Guía para el Trabajo Fin de Máster Curso 19-20 (Universidad de Málaga, 2019b). No lo encontraremos.

Durante el curso 2018/2019, como en el resto de cursos previos y posteriores, existía la posibilidad pública de presenciar las exposiciones de TFM; ejercido este derecho, en un tribunal concreto de TFM en la Universidad de Málaga, en base a lo que bajo mi humilde punto de vista fueron barbaridades pedagógicas de todo tipo -por parte de profesorado asociado de instituto, cuya formación pedagógica desconozco- y, en todo caso, en función de mi juicio pedagógico personal, a nada reflejado en la plantilla legal correspondiente (Universidad de Málaga, 2019a), presencié como se calificaba con un cinco a una alumna, con un trato por parte del tribunal hacia ella que provocó que saliera llorando tras lo que tuvo que oír. Las plantillas de evaluación, rúbricas y/o criterios de evaluación 
públicos deben emplearse en la corrección de los trabajos académicos, y los Trabajos de Fin de Máster deben estar fundamentados, ser personales, y han de defenderse ante un tribunal para obtener una calificación acorde a los citados documentos.

\section{LEGISLACIÓN $Y$ REQUISITOS DEL TRABAJO DE FIN DE MÁSTER}

Legislativamente, no encontramos una gran dedicación al MAES en el Estado español. Tres páginas en la Orden ECl/3858/2007, de 27 de diciembre, por la que se establecen los requisitos para la verificación de los títulos universitarios oficiales que habiliten para el ejercicio de las profesiones de Profesor de Educación Secundaria Obligatoria y Bachillerato, Formación Profesional y Enseñanzas de Idiomas; seis páginas en el Real Decreto 1834/2008, de 8 de noviembre, por el que se definen las condiciones de formación para el ejercicio de la docencia en la educación secundaria obligatoria, el bachillerato, la formación profesional y las enseñanzas de régimen especial y se establecen las especialidades de los cuerpos docentes de enseñanza secundaria. En el caso de la Universidad de Málaga, su Normativa sobre Trabajo Fin de Máster de la Facultad de Ciencias de la Educación (2016) establece distintas e interesantes opciones del mismo, pero su concreción (Universidad de Málaga, 2019b) establece un máximo de 75 páginas de extensión en el TFM, considerando que "constituye el Prácticum de este Máster" (ibídem, p. 1), estableciendo que la memoria del TFM debe contemplar contenidos concretos (ibíd.), entre los cuales se encuentran las finalidades y objetivos de la Educación Secundaria y/o Formación Profesional. De forma un tanto mecánica, considerando un número mínimo de páginas para la bibliografía de entre tres y cinco, tendríamos unas 70 páginas para los tres epígrafes que nos exige la Universidad de Málaga, es decir: Marco contextualizador de la profesión docente, diseño y fundamentación de un proyecto o plan de trabajo que refleje las competencias de la especialidad docente cursada, y reflexión crítica y valoración personal. Nunca habrá máximo de páginas en los anexos según la normativa del formato APA estándar elaborado por la Asociación Americana de Psicología. Si, aproximadamente, tendríamos 23 páginas por epígrafe, y en este primer epígrafe nos formulan 12 preguntas (ibíd.), tenemos casi dos páginas para contestar a cada pregunta. Los trabajos en general suelen -o tendrían quemantener una cierta compensación, bajo mi punto de vista. No tendría probablemente mucho sentido un epígrafe de cinco páginas, otro de 20 , y otro de 45 páginas; eso sí, habitualmente la reflexión crítica y valoración personal que podría encontrarse en los TFM suele tener una amplitud bastante reducida, a la par que acrítica: no es alto el número de alumnado que critica con contundencia el programa de formación del MAES y su desarrollo, al igual que alguien en un juicio no cuestiona a juez y fiscalía, por la cuenta que le trae. Pese a ello, hay quienes lo consideran un fraude (Romo, 2018), una estafa (Colegio Profesional de Periodistas de Andalucía, 2017), o el timo del tocomocho (Martí, 2010), como mínimo, a posteriori. En la enseñanza estatal española, existe la posibilidad de ser una persona completamente ignorante de casi todo; mientras se apruebe la enseñanza reglada, una carrera concreta, el MAES, y las oposiciones, con plaza, ya es posible ejercer la docencia, para toda la vida. Se premia la especialización y el presunto dominio de una asignatura concreta. Siguiendo el currículo que impone el Estado español, cuando no libros de texto en concreto, independientemente del mundo que nos rodea, el profesorado podría no sufrir inconveniente alguno (Nadal, 2015).

El Trabajo de Fin de Máster de la Universidad de Málaga nos obliga a responder a por qué y para qué educamos, utilizando referencias que apoyan los argumentos, la discusión y el 
análisis, con amplitud de la revisión bibliográfica, tanto general como de la especialidad y del tema escogido o hilo conductor, y con actualidad y relevancia didáctica de las referencias utilizadas, tanto generales como de la especialidad (Universidad de Málaga, 2019b). Todo ello, pese a que la mayoría (en base a las programaciones de los grados de los que provienen) del alumnado del Máster no estudió nada relacionado con enseñanza $y / o$ pedagogía hasta matricularse y comenzar el Máster, solo unos meses antes de entregar y defender el TFM. Tan actual y relevante didáctica y pedagógicamente es la formación del alumnado del MAES que suelen encontrarse habituales referencias a Miguel Ángel Santos Guerra (Universidad de Málaga, 2019d) y José Manuel Esteve Zarazaga (Universidad de Málaga, 2019e), es decir, a los mismos catedráticos que nos mencionaban como ideales hace más de dos décadas, y cuyas obras nos obligaban a leer.

Todo el material, actualizado o no, que nos haya proporcionado el profesorado del MAES, podremos referenciarlo, incluyendo, si fuera el caso, aquel material que dicho profesorado hubiera publicado. No lo olvidemos: dicho profesorado hay grandes posibilidades de que nos juzgue en el tribunal correspondiente que determinará que todos/as aquellos/as que se presenten son aptos/as para la enseñanza secundaria. $Y$ digo todos/as, por las cifras oficiales: en el caso de la Universidad de Málaga, más del $99,61 \%$ del alumnado del MAES aprueba, con lo cual, se roza casi a la perfección el aprobado general (Universidad de Málaga, 2019c). Estas cifras no son muy motivadoras para algunas profesionales que ejercemos la docencia o la tutorización en el Máster, y menos aún para quienes pretendemos realizar materiales docentes. No obstante, por coherencia personal, y más aún, por miedo fundado al profesorado que en el futuro podrían sufrir menores de edad, deseando que no sea como el que personalmente sufrí, es fundamental para que ningún tribunal pueda, a mi entender, cuestionar lo que escribimos, formarnos de forma crítica en pedagogía.

Lo primero, la ley. Estemos de acuerdo, o no, nos guste o no, es lo que rige el sistema de enseñanza del Estado español. No entraremos en las entidades internacionales que, a mi juicio, ejercen de titiriteros/as. Por tanto, tenemos a nuestra disposición todas las normativas que nos sean útiles. En primer lugar, la ley general, en este caso, y para cuando escribo esto, la gloriosa Ley Orgánica 8/2013, de 9 de diciembre, para la mejora de la calidad educativa (LOMCE). El mundo idílico que nos plantea se encuentra en su preámbulo, y las primeras ocho líneas de dicho texto consideran que

El alumnado es el centro y la razón de ser de la educación. El aprendizaje en la escuela debe ir dirigido a formar personas autónomas, críticas, con pensamiento propio. Todos los alumnos y alumnas tienen un sueño, todas las personas jóvenes tienen talento. Nuestras personas y sus talentos son lo más valioso que tenemos como país. Por ello, todos y cada uno de los alumnos y alumnas serán objeto de una atención, en la búsqueda de desarrollo del talento, que convierta la educación en el principal instrumento de movilidad social, ayude a superar barreras económicas y sociales y genere aspiraciones y ambiciones realizables para todos (p. 97858).

Si queremos responder de forma sublime a para qué y por qué educar, aquí tenemos una fuente primaria que nos deleita. Disponemos de nada más y nada menos que casi nueve páginas en el preámbulo de la ley de las cuales obtener todo el discurso y la verborrea del régimen. Sin embargo, no ignoremos que "planifican aberraciones pedagógicas o que se desarrollan sobre el error" (De la Herrán, 2020, p. 44), por no entrar en la historia del Estado español o en la formación socio-pedagógica de los/las legisladores/as. 


\section{CONCLUSIONES}

Hay una palabra clave que hemos de entender, básica en función de la legislación vigente; no nos detendremos en este escrito en considerar de lo que hablamos, las competencias, como una mistifación pedagógica (Hirtt, 2009). Como era de esperar, no es un concepto ibérico precisamente, y lo vamos a obtener, como siempre que podamos, de fuente primaria, en este caso, del inicio (diría preámbulo, pero no recibe nombre alguno) del Real Decreto 1105/2014, de 26 de diciembre, por el que se establece el currículo básico de la Educación Secundaria Obligatoria y del Bachillerato, una disposición que no debiera faltar en ningún TFM:

En línea con la Recomendación 2006/962/CE del Parlamento Europeo y del Consejo, de 18 de diciembre de 2006, sobre las competencias clave para el aprendizaje permanente, este real decreto se basa en la potenciación del aprendizaje por competencias, integradas en los elementos curriculares para

propiciar una renovación en la práctica docente y en el proceso de enseñanza y

aprendizaje. Se proponen nuevos enfoques en el aprendizaje y evaluación, que han d e suponer un importante cambio en las tareas que han de resolver los alumnos y planteamientos metodológicos innovadores. La competencia supone una combinación de habilidades prácticas, conocimientos, motivación, valores éticos, actitudes, emociones, y otros componentes sociales y de comportamiento que se movilizan conjuntamente para lograr una acción eficaz. Se contemplan, pues, c o mo conocimiento en la práctica, un conocimiento adquirido a través de la participación activa en prácticas sociales que, como tales, se pueden desarrollar tanto en el contexto educativo formal, a través del currículo, como en los contextos educativos no formales e informales. Las competencias, por tanto, se conceptualizan como un «saber hacer» que se aplica a una diversidad de contextos académicos, sociales y profesionales. Para que la transferencia a distintos contextos sea posible resulta indispensable una comprensión del conocimiento presente en las competencias, y la vinculación de éste con las habilidades prácticas 0 destrezas que las integran (p. 5).

Contamos con dos fuentes primarias fundamentales, y podemos añadir, con nuestra propia narrativa, un análisis de objetivos de la Enseñanza Secundaria Obligatoria (ESO) y Bachillerato que incluye el Real Decreto mencionado. Eso sí, la plantilla de evaluación de la Universidad de Málaga nos pide literalmente "justificación y sentido que el marco contextualizador de la profesión docente y el tema escogido da [sic] al proyecto o plan de trabajo en el marco de la especialidad cursada" (Universidad de Málaga, 2019a, p. 1). Lo que personalmente entiendo de dicha frase estaría relacionado con que lo que escribamos en este sub-apartado esté relacionado con el proyecto de intervención que hayamos desarrollado, es decir, que si escribimos que educamos para formar personas autónomas, críticas, y con pensamiento propio, por ejemplo, luego no presentaremos una intervención en base a una secuencia didáctica que supondría un aprendizaje que no generara tal tipo de personas; relacionar competencias, igualmente, sería necesario.

Una revisión crítica y presentación/utilización de síntesis de los trabajos más relevantes suele ser una exigencia. Aunque en modo alguno estoy de acuerdo, esta relevancia puede considerarse el Ranking Scholarly Publishers Indicators. En este caso, podemos buscar libros que teoricen sobre para qué y por qué educamos de las editoriales mejor valoradas, si es que deseamos cumplir con lo que nos exigen. Algo similar sucede con las revistas científicas de impacto sobre educación (en WoS y JCR / Scopus y 
SJR). Otro nuevo ranking. Aunque fuera el mismo texto, lo que escribiera, por ejemplo, una catedrática de pedagogía en un blog, o en un periódico, o en una web cualquiera, no tendría la misma presunta relevancia que si publicara literalmente lo mismo en una editorial o revista de alto impacto. En definitiva, debemos tener claro usar referencias legislativas (no solo las aportadas aquí brevemente: tenemos más normativas estatales y por supuesto autonómicas) y bibliografía con la cual apoyar lo que redactemos sobre para qué y por qué educar. Y por supuesto, aportar ideas propias.

Los porqués que deseemos establecer estarán en función de factores fundamentales, desde nuestra cosmovisión del mundo, hasta las opciones que nos permita el Estado, salvo que vayamos más allá. Para autores/as afines al régimen, hay que salvar a la infancia de la radicalización, como expresa el catedrático de filosofía de instituto, Doctor Honoris Causa por la Universidad Politécnica de Valencia y autor de decenas de libros Jose Antonio Marina (2016). De esta forma, con esta ideología, es posible, como fue su caso, lograr ser asesor educativo del partido que esté en el gobierno, como fue su caso con el Partido Popular español (Montesinos, 2015; Sanmartín, 2015). Para otros autores, la radicalización es positiva, porque es preponderantemente crítica, amorosa, humilde y comunicativa (Freire, 1973).

Probablemente no encontraremos obstáculos si deseamos vigilar y castigar (Foucault, 2003), si nuestra opción fuera por encajar en los engranajes del sistema. Nadar contracorriente, configurar una didáctica con el objetivo de lograr una educación para la evolución de la vida humana y planetaria (Arboleda, 2020) no es sencillo, y en ocasiones puede ocasionar despidos (Wilson, 2005). Ya sea laboralmente, o en un trabajo escrito como el TFM, por qué y para qué educar probablemente es una decisión que debemos fundamentar, con un planteamiento epistemológico y una opción personal en sí conocedora del contexto en el que nos encontramos y en cuyas manos, salvo revolución, nos encontraremos.

\section{REFERENCIAS BIBLIOGRÁFICAS}

Arboleda, J. C. (2020). Educar para la evolución de la vida humana y planetaria. Una perspectiva comprensivo edificadora. Revista Boletín Redipe, Vol. 9, №. 6, 2020, págs. 51-65.

Colegio Profesional de Periodistas de Andalucía. (2017). El CPPA considera una "estafa» que las universidades públicas andaluzas oferten a los periodistas un máster que no les servirá para nada. Recuperado de https:// periodistasandalucia.es/el-cppa-considerauna-estafa-que-las-universidades-publicasandaluzas-oferten-a-los-periodistas-un-masterque-no-les-servira-para-nada/

Chicote, J. (2020, febrero 3). Condenados por plagio un catedrático y un profesor de la universidad de Alcalá. Abc España. Recuperado 20 junio 2020, de https://www.abc.es/espana/ abci-condenados-plagio-catedratico-y-profesoruniversidad-alcala-202002030251_noticia.html

De la Herrán, A. (2020). ¿Por qué los currícula no pueden educar? Una mirada radical inclusiva. Revista Boletín Redipe, Vol. 9, № . 6, 2020, págs. 42-50.

Delgado, E. (2020, enero 13). La trama de las tesis plagiadas en la Camilo José Cela se extiende a la Universidad de Málaga. Abc España. Recuperado 20 junio 2020, de https:// www.abc.es/espana/abci-trama-tesis-plagiadascamilo-jose-cela-extiende-universidadmalaga-202001130124_noticia.html

Foucault, M. (2003). Vigilar y castigar. Buenos Aires, República Argentina: Siglo XXI Editores. 
Freire, P. (1973). La educación como práctica de la libertad. Buenos Aires, República Argentina: Siglo XXI Editores.

Hirtt, N. (2009). El planteamiento por competencias: una mistificación pedagógica. L'école démocratique, № 39, 2009, págs. 1-15.

Ley Orgánica 8/2013, de 9 de diciembre, para la mejora de la calidad educativa. «BOE», núm. 295, de 10 de diciembre de 2013, páginas 97858 a 97921. Recuperado de http://www.boe.es/ buscar/doc.php?id=BOE-A-2013-12886 [Fecha de consulta: 29 de junio de 2020].

Marina, J. A. (2016). Calidad y equidad: un viejo debate que no puede ser caricaturizado [Entrada blog]. Recuperado de https://www. joseantoniomarina.net/articulo/

Martí, J. (2010). El máster de formación del profesorado, o el timo del tocomocho [Entrada blog]. Recuperado de https://xarxatic.com/elmaster-de-formacion-del-profesorado-o-el-timodel-tocomocho/

Montesinos, O. R. (2015). José Antonio Marina y el PP [Entrada blog]. Recuperado de http:// lacuevadelgigante.blogspot.com/2015/11/joseantonio-marina-y-el-pp.html

Moreno, R. (2020, enero 24). El Supremo confirma la condena a un profesor de universidad por copiar a un alumno su trabajo de investigación. Confilegal. Recuperado 20 junio 2020, de https://confilegal.com/20200124el-supremo-confirma-la-condena-a-un-profesorde-universidad-por-copiar-a-un-alumno-sutrabajo-de-investigacion/

Nadal, A. (2015). Análisis y valoración de la vigencia de los principios pedagógicos de la Escuela Moderna de Francisco Ferrer Guardia en el Estado español en el siglo XXI. Estudio de casos (Tesis doctoral. Universidad de Málaga, Andalucía, Estado español). Recuperado de http://hdl.handle.net/10630/10412
Orden ECl/3858/2007, de 27 de diciembre, por la que se establecen los requisitos para la verificación de los títulos universitarios oficiales que habiliten para el ejercicio de las profesiones de Profesor de Educación Secundaria Obligatoria y Bachillerato, Formación Profesional y Enseñanzas de Idiomas. «BOE» núm. 312, de 29 de diciembre de 2007, páginas 53751 a 53753. Recuperado de https://www.boe.es/ diario_boe/txt.php?id=BOE-A-2007-22450 [Fecha de consulta: 29 de junio de 2020].

Ramajo, J. (2020, febrero 9). El Tribunal Supremo castiga a una profesora acusada de plagios reincidentes por causar "desprestigio a la Universidad". eldiario.es. Recuperado 20 junio 2020, de https://www.eldiario. es/andalucia/sevilla/Universidad-Sevillaplagios_0_989701419.html

Real Decreto 1105/2014, de 26 de diciembre, por el que se establece el currículo básico de la Educación Secundaria Obligatoria y del Bachillerato. «BOE» núm. 3 , de 3 de enero de 2015, páginas 1 a 35. Recuperado de https:// www.boe.es/buscar/act.php?id=BOE-A-2015-37

Real Decreto 1834/2008, de 8 de noviembre, por el que se definen las condiciones de formación para el ejercicio de la docencia en la educación secundaria obligatoria, el bachillerato, la formación profesional y las enseñanzas de régimen especial y se establecen las especialidades de los cuerpos docentes de enseñanza secundaria. «BOE» núm. 287, de 28 de noviembre de 2008, páginas 47586 a 47591 . Recuperado de https://www.boe.es/buscar/doc. php?id=BOE-A-2008-19174 [Fecha de consulta: 29 de junio de 2020].

Romo, G. (2018, abril 9). El fraude del Máster de Formación de Profesorado [Entrada blog]. Recuperado de http://toomatesbloc.blogspot. com/2018/04/el-fraude-del-master-deformacion-de.html 
Sanmartín, O. R. (2015, noviembre 3). José Antonio Marina: 'El buen maestro no puede cobrar lo mismo que el malo'. El Mundo. Recuperado 20 junio 2020, de https://www. elmundo.es/sociedad/2015/11/03/5637c295268 e3ecf698b4691.html

Significados.com. (2020). Significado de Educar. Recuperado de https://www.significados.com/ educar/ [Fecha de consulta: 10 de junio de 2020].

Universidad de Málaga (2016). Normativa sobre Trabajo Fin de Máster de la Facultad de Ciencias de la Educación. Recuperado de https://www. uma.es/master-en-profesorado/cms/menu/plande-estudios/tfm/ [Fecha de consulta: 29 de junio de 2020].

Universidad de Málaga (2019a). Plantilla de Evaluación de la Comisión Evaluadora. Recuperado de https://www.uma.es/master-enprofesorado/cms/menu/plan-de-estudios/tfm/ [Fecha de consulta: 29 de junio de 2020].

Universidad de Málaga (2019b). Guía para el Trabajo Fin de Máster Curso 2019 - 2020. Recuperado de https://www.uma.es/master-enprofesorado/cms/menu/plan-de-estudios/tfm/ [Fecha de consulta: 29 de junio de 2020].

Universidad de Málaga (2019c). Resultados del título. Recuperado de https://www.uma.es/ master-en-profesorado/cms/menu/informaciongeneral/ [Fecha de consulta: 29 de junio de 2020].

Universidad de Málaga (2019d). Guía Docente de la Asignatura. Curso 2019 / 2020. Procesos y contextos educativos. Recuperado de https://oas.sci.uma.es:8443/ht/2019/ ProgramasAsignaturas_Titulacion_5139_ AsigUMA_101352.pdf [Fecha de consulta: 29 de junio de 2020].

Universidad de Málaga (2019e). Guía Docente de la Asignatura. Curso 2019 / 2020.
Sociedad, familia y educación. Recuperado de https://oas.sci.uma.es:8443/ht/2019/ ProgramasAsignaturas_Titulacion_5139_ AsigUMA_101355.pdf

Villarreal, A., Escudero, J. y Méndez, R. (2019, octubre 14). La churrería de tesis de la Camilo: familiares y plagiadores para sacar 26 en tres años. El Confidencial. Recuperado 20 junio 2020, de https://www.elconfidencial.com/ espana/2019-10-14/universidad-camilo-josecela-plagios-tesis-doctores_2280575/

Wilson, J. (2005, octubre 25). Protests at Yale over sacking of rebel professor. The Guardian. Recuperado 20 junio 2020, de https://www. theguardian.com/world/2005/oct/25/usa. internationaleducationnews 